\title{
Investigation of the sliding behavior between steel and mortar for seismic applications in structures
}

\author{
Jason McCormick $1, *, \dagger, \ddagger$, Takuya Nagae ${ }^{2, \S}$, Masahiro Ikenaga $a^{3, \mathbb{T}}$, \\ Peng-Cheng Zhang ${ }^{4, \|}$, Mika Katsuo ${ }^{3, \mathbb{I}}$ and Masayoshi Nakashima ${ }^{3, * *}$ \\ ${ }^{1}$ Department of Civil and Environmental Engineering, University of Michigan, Ann Arbor, MI 48109-2125, U.S.A. \\ ${ }^{2}$ National Research Institute for Earth Science and Disaster Prevention, Miki-shi, Hyogo-ken 673-0515, Japan \\ ${ }^{3}$ Disaster Prevention Research Institute, Kyoto University, Kyoto 611-0011, Japan \\ ${ }^{4}$ School of Architecture and Civil Engineering, Xiamen University, Fujian Province 361005, China
}

\begin{abstract}
SUMMARY
The friction developed between a steel base plate and a mortar base contributes shear resistance to the building system during a seismic event. In order to investigate the possible sliding behavior between the base plate and the mortar, a shake table study is undertaken using a large rigid mass supported by steel contact elements which rest on mortar surfaces connected to the shake table. Horizontal input accelerations are considered at various magnitudes and frequencies. The results provide a constant friction coefficient during sliding with an average value of approximately 0.78 . A theoretical formulation of the friction behavior is also undertaken. The theoretical equations show that the sliding behavior is dependent on the ratio of the friction force to the input force. The addition of vertical accelerations to the system further complicates the sliding behavior as a result of the varying normal force. This results in a variable friction resistance which is a function of the amplitude, phase, and frequency of the horizontal and vertical input motions. In general, this study showed a consistent and reliable sliding behavior between steel and mortar. Copyright (C) 2009 John Wiley \& Sons, Ltd.
\end{abstract}

Received 11 July 2008; Revised 20 January 2009; Accepted 20 January 2009

KEY WORDS: friction; steel; mortar; steel structures; shake table tests; seismic

\footnotetext{
*Correspondence to: Jason McCormick, Department of Civil and Environmental Engineering, University of Michigan, Ann Arbor, MI 48109-2125, U.S.A.

${ }^{\dagger}$ E-mail: jpmccorm@umich.edu

$\ddagger$ Assistant Professor.

$\S$ Research Fellow.

"Graduate Student.

"Associate Professor.

** Professor.
}

Contract/grant sponsor: Grant-in-Aid for Scientific Research; contract/grant number: 18760419

Contract/grant sponsor: Chinese Government Supported Researchers Program

Contract/grant sponsor: Japan Society for the Promotion of Science Postdoctoral Fellowship for Foreign Researchers Program

Copyright (C) 2009 John Wiley \& Sons, Ltd. 


\section{INTRODUCTION}

Steel structural systems are widely used in seismically active areas around the world. Often in building systems, these structures are constructed as either moment-resisting frames or special concentrically braced frames to provide the lateral force resistance necessary to resist seismic loads. As a result of damage sustained during past earthquakes, performance-based design philosophies, and strong column-weak beam requirements, much of the previous research on the behavior of steel structures has focused on the beam hinging mechanism in steel moment frames and more recently brace yielding in special concentrically braced frames. Only a limited amount of work has focused on column base behavior and current design methods for column base systems [1-4]. This has led to exposed base plate-type column bases (or standard base-plate connections) often being designed as fixed connections with the base plate being connected to the foundation through anchor bolts in order to resist shear forces and moments during a seismic event. These bolted column base systems are often employed because of ease of construction and economic efficiency [1-4].

The current design standards for column base systems acknowledge three primary means of shear transfer [5]. The first means is friction between the steel base plate and the mortar or concrete surface on which it rests. In this case, the friction coefficient is often taken as 0.55 for steel on mortar and 0.7 for steel on concrete [5]. The second means of shear transfer is bearing against concrete of the base plate, column, and/or shear lug. The last means of shear transfer is shear sustained by the anchor rods. Friction is often considered as an initial means of resisting shear until sliding begins, but many building codes for seismic regions in the United States do not allow for the consideration of friction when resisting code specified earthquake loads [6]. Alternatively, Japanese design codes acknowledge the shear resistance gained from both friction and anchor bolt bearing for exposed column bases. However, only the smaller of these two values is considered for design purposes so that the combined effect is not utilized [7].

Consideration of friction resistance in column base design for seismic regions has been limited due to a lack of information in regards to the sliding behavior of steel on either a concrete or mortar surface. There have been few studies on friction behavior, which have mainly focused on rigid body sliding under earthquake motions [8-12], whereas many other studies within structural engineering have focused on base isolation systems [13-18] and energy dissipation systems [19-22] that do not consider traditional structural materials. A recent study conducted in Japan did compare the static friction coefficient between steel and steel to that between steel and mortar. The results showed friction coefficient values to be large enough and consistent enough to consider in column base design in seismically active regions [23]. However, this study did not consider the dynamic sliding behavior which would be expected during an earthquake. If a predictable and reliable friction behavior can be obtained for dynamic sliding between a steel base plate and the mortar surface on which it rests, it may be applicable to superimpose the friction force with other shear-resisting mechanisms in the column base, such as anchor bolt shear, resulting in a more economic and practical design. A possible inhibiting factor in considering friction for column base seismic design is vertical loading effects since the friction force is dependent on the vertical force between the two surfaces. These effects may limit the applicability of considering friction behavior to low-to-mid rise structures where the overturning moments and vertical loads tend to be smaller.

In order to address this lack of knowledge with respect to the dynamic sliding behavior of steel on mortar, a shake table study is carried out. Both horizontal-only and combined horizontal and vertical loadings are considered to simulate the sliding behavior of a steel column base. The sliding behavior is also considered from a theoretical point of view in order to better understand the nature 
and mechanism of the sliding behavior. The knowledge obtained in regards to the dynamic sliding behavior of steel on mortar may provide a means of improving column base design in the future. A secondary benefit of sliding may be the development of a new damping system taking advantage of sliding of steel on mortar if the friction behavior can be shown to be stable and robust.

\section{EXPERIMENTAL STUDY}

Only a limited number of studies in the past have examined the sliding behavior between mortar and steel, even though these materials are two of the most commonly used in building construction. A previous study in Japan explored the static friction coefficient between various steel surfaces and mortar. The average friction coefficients considering the various types of steel surfaces were between 0.52 and 0.97 , where the larger values are approximately twice those typically associated with steel sliding on steel [23]. However, consistency of the friction coefficient and sliding behavior under dynamic motion is also necessary to improve upon current column base design procedures. In order to further this work, an experimental study was conducted to evaluate the dynamic sliding properties of steel on mortar.

\subsection{Experimental test setup}

A shake table study was conducted to determine the fundamental sliding characteristics between steel and mortar under dynamic loads. The six degree of freedom shake table located at the Disaster Prevention Research Institute of Kyoto University in Japan has plan dimensions of $3 \mathrm{~m} \times 5 \mathrm{~m}$. The shake table can provide a maximum acceleration and velocity of $1.0 \mathrm{~g}$ and $1.5 \mathrm{~m} / \mathrm{s}$ in each direction, respectively. All shaking was performed in the direction parallel to the long dimension of the shake table both with and without vertical motion included. The test specimen consisted of three main elements: the mortar surface, the steel friction surface, and the rigid mass as can be seen in Figure 1. In order to roughly simulate four column bases of a building structure, the test specimen had four points of contact for sliding.

The four mortar surfaces were cast on top of Japanese steel H-Sections (similar to U.S. Wshapes) and secured to the H-sections using embedded steel studs. Steel angles attached to the $\mathrm{H}$-section were used to border the mortar surface to provide a stable and immobile block. The $\mathrm{H}$-sections were the only portion of the test specimen that was directly bolted to the shake table. A commercial mortar was used with a composition of $46 \%$ high, early strength Portland cement, $50 \%$ fine aggregate, and $4 \%$ admixture. The dimensions of the mortar surface were $550 \mathrm{~mm} \times 1020 \mathrm{~mm}$ allowing for approximately $300 \mathrm{~mm}$ of sliding in either direction when the steel surface is placed at the center of the mortar surface. A smooth and level surface was obtained by placing a polished steel plate on top of the wet mortar and leaving it in place while the mortar set. This procedure was used to represent the mortar conditions under a steel column base as constructed in Japan. The same four mortar surfaces were used throughout the study with only the removal of accumulated debris between subsequent tests. Material tests showed that the mortar had a compressive strength of approximately $35 \mathrm{MPa}$ at the time of testing.

The steel friction elements consisted of $250 \mathrm{~mm} \times 250 \mathrm{~mm} \times 60 \mathrm{~mm}$ blocks of steel, which were tapered on one side down to a $75 \mathrm{~mm} \times 75 \mathrm{~mm}$ square. This smaller square had a polished surface and represented the contact area between the steel and mortar. A polished surface was chosen to represent the bottom of an unpainted column base. Other steel surfaces, such as mill 

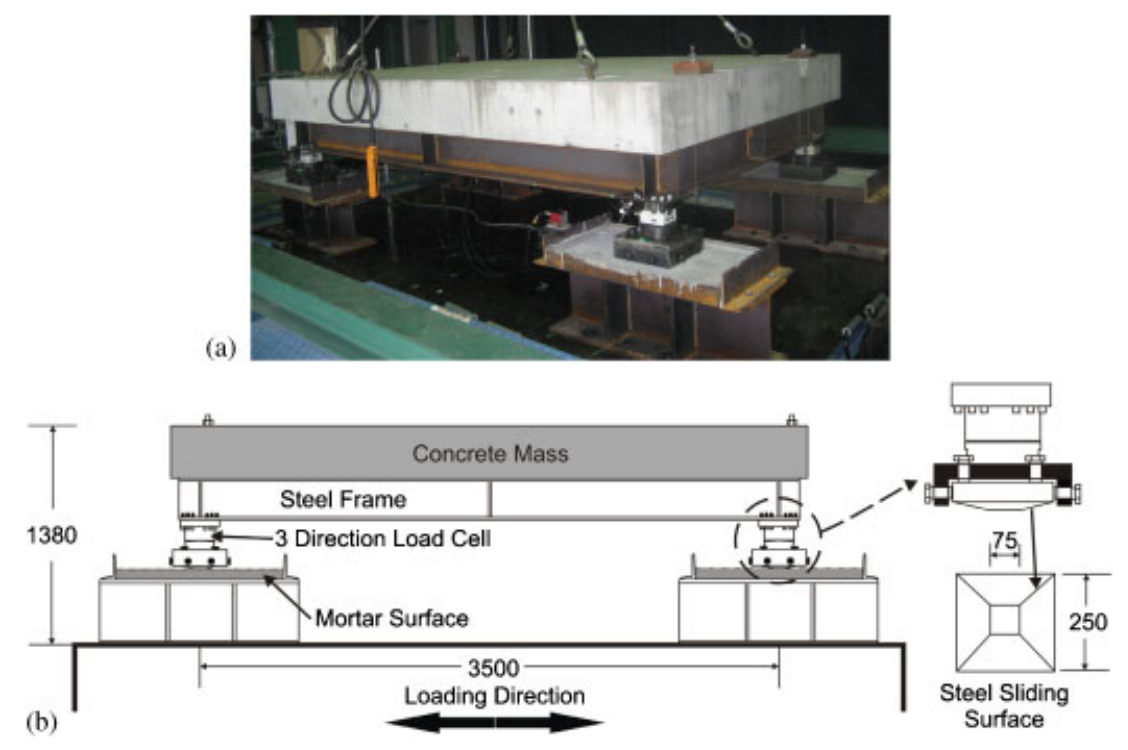

Figure 1. (a) Photograph of the test specimen and (b) specimen details (all dimensions in $\mathrm{mm}$ ).

scale, will result in a change of the friction coefficient and are being considered in later studies. The steel friction element was held in place beneath the load cells and connected to the rigid mass. Vertical and horizontal bolts allowed for adjustment of the slope of the steel friction surface to ensure full contact with the mortar and equal distribution of the gravity load between each of the four points of contact.

The rigid mass consisted of a $68.6 \mathrm{kN}$ concrete block sitting on top of a $9.2 \mathrm{kN}$ horizontal steel frame. The concrete mass was connected to the steel frame using post-tensioning rods at four points. Combining the weight of the concrete block, steel frame, load cells, and steel friction blocks provided a total specimen weight of $82.0 \mathrm{kN}$. This overall weight results in a pressure of $14.6 \mathrm{MPa}$ on the mortar surface at the contact points. This pressure is below the maximum allowable bearing pressure for steel base plates resting on mortar surfaces and would be on the lower side of that experienced by a low-rise steel building frame. Further details and dimensions of the test specimen can be seen in Figure 1(b).

The test specimen was instrumented with tri-axial load cells to measure both the vertical load at each contact point and the shear force generated by the contact between the steel and mortar. Laser displacement transducers were used to measure the absolute displacements of both the mortar surface and rigid frame, whereas strain potentiometers were used to directly measure the relative sliding between the two. Accelerometers were also installed on the concrete mass and on the surface of the shake table in order to obtain both relative and absolute acceleration values.

\subsection{Loading protocol}

The shake table was run in acceleration control using a sinusoidal input wave. The sinusoidal wave was gradually ramped up to its intended maximum input acceleration during the first couple of cycles and then oscillated at this level for a total duration of $10 \mathrm{~s}$. For the first set of tests, 
Table I. Loading protocol matrix for horizontal-only sinusoidal input acceleration tests.

\begin{tabular}{lccc}
\hline & \multicolumn{3}{c}{ Frequency $(\mathrm{Hz})$} \\
\cline { 2 - 4 } Maximum acceleration $\left(\mathrm{m} / \mathrm{s}^{2}\right)$ & 1 & 2 & 5 \\
\hline 6 & $1-6$ & $2-6$ & $5-6$ \\
7 & $1-7$ & $2-7$ & $5-7$ \\
8 & $1-8$ & $2-8$ & $5-8$ \\
9 & $1-9$ & $2-9$ & $5-9$ \\
\hline
\end{tabular}

only horizontal accelerations were applied parallel to the long direction of the test specimen. Three different loading frequencies were considered: 1, 2, and $5 \mathrm{~Hz}$. For each of these loading frequencies, tests were run at increasing acceleration magnitudes between 6 and $9 \mathrm{~m} / \mathrm{s}^{2}$. For the second set of tests, both horizontal and vertical accelerations were applied simultaneously in order to consider the effect of varying vertical forces on the friction behavior. The vertical accelerations were applied with a $3 \mathrm{~m} / \mathrm{s}^{2}$ acceleration magnitude at either 1,2 , or $5 \mathrm{~Hz}$ and in-phase with the horizontal motion that was applied with the same loading frequencies and acceleration magnitudes as the previous horizontal-only tests. However, limitations of the shake table prevented some of the larger magnitude tests from being run. Table I provides the loading protocol test matrix for the horizontal-only sinusoidal input wave tests. A third set of tests were conducted to evaluate the effects of non-stationary motion on the sliding behavior. To simulate non-stationary motion, the JMA Kobe ground motion was adopted.

\subsection{Horizontal loading test results}

For each of the horizontal loading tests, an acceleration magnitude was specified for the input sinusoidal wave. This acceleration magnitude along with the input frequency is used throughout the remainder of this paper to specify each individual test. However, it should be noted that the actual maximum acceleration value recorded by the accelerometer attached to the shake table tended to vary somewhat from this value. This difference was attributed to the control difficulties associated with the large mass of the test specimen and high acceleration values being used. In light of the objectives of this study, this difference was not significant as long as the actual accelerations on the table were recorded accurately.

Figure 2 provides the relative displacement time histories and friction coefficient versus relative displacement results for the specimen undergoing the $9 \mathrm{~m} / \mathrm{s}^{2}$ magnitude sinusoidal input wave run at 1,2 , and $5 \mathrm{~Hz}$, respectively. The displacement results are obtained by averaging the relative displacement values for all four contact surfaces. Since the specimen was rigid, there was very little difference between the relative displacement values measured at each contact point. The friction coefficient was obtained by summing the shear force measured by all four load cells and dividing this value by the sum of the normal force measured by all four load cells. An example of the steel and mortar sliding surfaces is shown in Figure 3.

All three loading frequencies display a similar sliding behavior with an overall increase in the relative displacement in one direction as shown in Figure 2(a), (c), and (e). Owing to the initial ramping up to the maximum acceleration level, the behavior of the initial cycles is slightly different, but does stabilize after the first $5 \mathrm{~s}$ of loading. Thus, the last $5 \mathrm{~s}$ of loading will be used to 

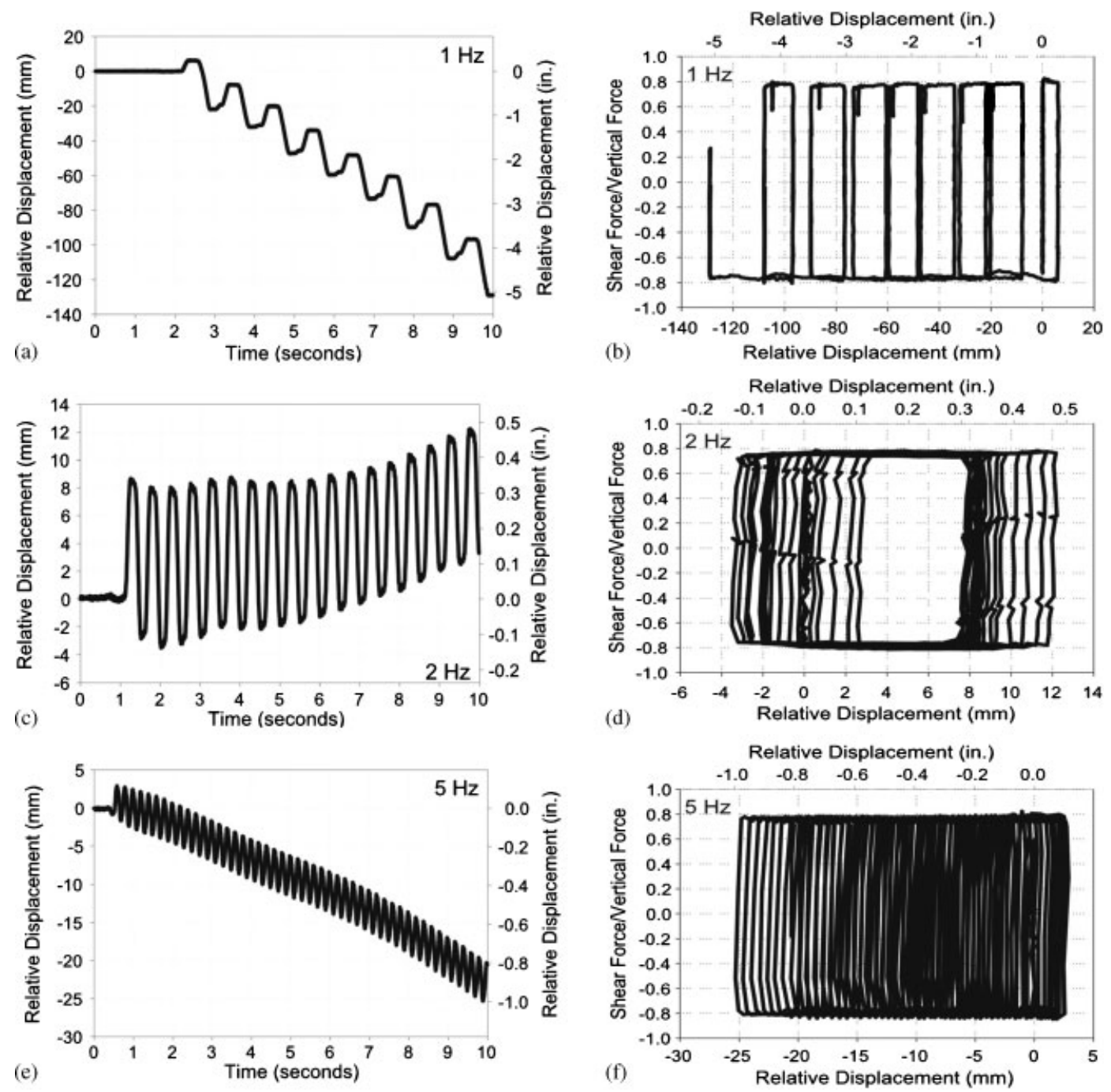

Figure 2. (a, c, e) Relative displacement time history and (b, d, f) friction coefficient versus relative displacement results for the $9 \mathrm{~m} / \mathrm{s}^{2}$ loading run at 1,2 , and $5 \mathrm{~Hz}$, respectively.

further analyze the results. For all three loading frequencies, sliding did not initiate until the $7 \mathrm{~m} / \mathrm{s}^{2}$ acceleration level. Larger accelerations resulted in an increase in the sliding distance for individual cycles, but did not show any specific trends with respect to the overall relative displacement of the specimen. The final overall relative displacements were measured as 135,3 , and $26 \mathrm{~mm}$ for the 1 , 2 , and $5 \mathrm{~Hz}$ loading frequencies, respectively. Although the overall relative displacement showed no trends with respect to loading frequency, the amplitude of each individual cycle did decrease with an increase in the loading frequency from approximately $20 \mathrm{~mm}$ at $1 \mathrm{~Hz}$ to approximately $6 \mathrm{~mm}$ at $5 \mathrm{~Hz}$. 


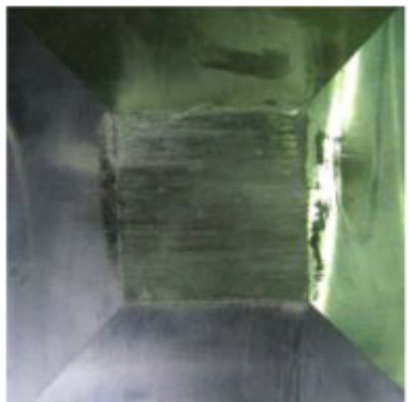

(a)

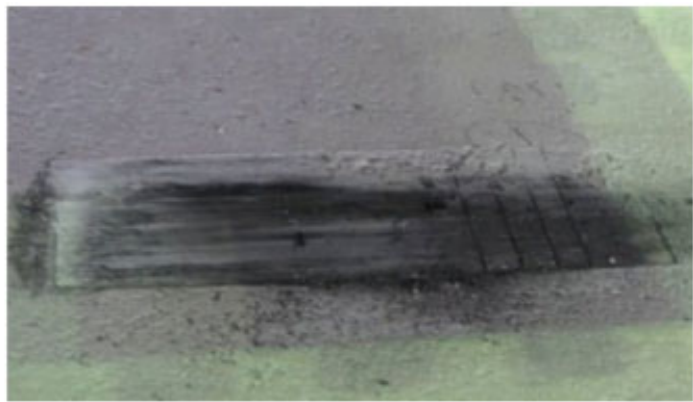

(b)

Figure 3. (a) Steel and (b) mortar sliding surfaces.

Table II. Average maximum measured friction coefficients.

\begin{tabular}{lccc}
\hline & \multicolumn{3}{c}{ Frequency $(\mathrm{Hz})$} \\
\cline { 2 - 4 } Maximum acceleration $\left(\mathrm{m} / \mathrm{s}^{2}\right)$ & 1 & 2 & 5 \\
\hline 6 & $-\overline{-}$ & $-\bar{c}$ & - \\
7 & 0.76 & 0.75 & 0.81 \\
8 & 0.77 & 0.78 & 0.79 \\
9
\end{tabular}

Figure 2(b), (d), and (f) show a consistent value for the friction coefficient throughout each loading. The average maximum friction coefficients during sliding varied between 0.76 and 0.82 with an overall average value for all tests being 0.78 . Table II provides the average maximum friction coefficient for each individual test. In general, the friction coefficient was approximately the same regardless of the acceleration level and loading frequency. The stability of the friction coefficient during the large number of loading cycles appears to be unique to steel sliding on mortar as studies of steel friction dampers often show a significant degradation of the friction coefficient due to surface wear and temperature change during cycling. This result suggests the possibility of implementing steel on mortar sliding systems for damping applications given the consistent behavior over a large number of cycles.

The relative velocity of the specimen increased significantly with an increase in the loading acceleration. The maximum relative velocities were measured as 140,110 , and $90 \mathrm{~mm} / \mathrm{s}$ for the 1 , 2 , and $5 \mathrm{~Hz}$ loading frequencies, respectively. At these velocities, the friction coefficient appears to be constant during sliding as can be seen in Figure 2(b), (d), and (f).

\section{THEORETICAL FORMULATION FOR SLIDING BEHAVIOR}

In light of the experimental results, a theoretical formulation of the sliding behavior between two materials is undertaken to better capture the nature and governing mechanisms of the sliding. This is done by representing the steel contact surface as a rigid block of mass, $m$, and the mortar 
surface as the horizontal plane on which the rigid block rests. The system is excited by having the horizontal plane on which the block rests undergo a horizontal sinusoidal motion to simulate the movement of the shake table or an actual earthquake. The block is assumed to be a point load, and thus does not provide any added overturning moment. In general, the sliding behavior can be separated into four cases based on the ratio of the friction force, $R$, to the amplitude of the input force (mass multiplied by the horizontal acceleration), $F$. The friction force is defined as

$$
R=\mu \cdot m \cdot g
$$

where $\mu$ is the friction coefficient and $g$ is the gravitational constant.

All formulations are developed considering that the system is initially at rest at $t_{0}$ and the friction coefficient, $\mu$, is constant (i.e. the static and dynamic friction coefficient values are considered the same). The formulation is done in a piecewise manner to account for the fact that the friction force changes direction based on the direction of the sliding motion.

\subsection{Case I: no sliding}

The governing equation of motion for the defined system can be written as

$$
m \ddot{y}(t) \pm R=F \sin (\omega t)
$$

where $\ddot{y}(t)$ is the relative acceleration of the block and $\omega$ is the frequency of the sinusoidal input wave in radians per second.

The block will not experience sliding until the input force overcomes the friction force as can be seen in Figure 4(a). Thus, Case I is defined as the case in which the block does not undergo sliding and occurs when $R / F>1.0$.

\subsection{Case II: equal sliding in both directions}

Provided that the $R / F$ ratio is less than 1.0, the block will slide with respect to the surface on which it rests during the input motion. For this case, sliding initiates at time, $t_{1}$, when the input force is equal to the friction force as defined in Equation (3) and depicted in Figure 4(b-d).

$$
\omega t_{1}=\arcsin \left(\frac{R}{F}\right)
$$

When sliding initiates, the relative acceleration of the block can be determined by solving the governing equation of motion. The relative velocity and displacement are obtained by single and double integration of the acceleration equation with respect to time.

$$
\begin{aligned}
\ddot{y}_{1}(t)= & \frac{F}{m} \sin (\omega t)-\frac{R}{m}>0 \text { for }\left[\omega t_{1}<\omega t<\omega t_{3}\right] \\
\dot{y}_{1}(t)= & \frac{-F}{m \omega}\left[\left[\cos (\omega t)-\cos \left(\omega t_{1}\right)\right]+\frac{R}{F}\left(\omega t-\omega t_{1}\right)\right] \quad \text { for }\left[\omega t_{1}<\omega t<\omega t_{3}\right] \\
y_{1}(t)= & \frac{F}{m \omega^{2}}\left\{-\left[\sin (\omega t)-\sin \left(\omega t_{1}\right)\right]-\frac{R}{2 F}\left[(\omega t)^{2}-\left(\omega t_{1}\right)^{2}\right]\right. \\
& \left.+\cos \left(\omega t_{1}\right) \cdot\left(\omega t-\omega t_{1}\right)+\frac{R}{F} \omega t_{1}\left(\omega t-\omega t_{1}\right)\right\} \quad \text { for }\left[\omega t_{1}<\omega t<\omega t_{3}\right]
\end{aligned}
$$




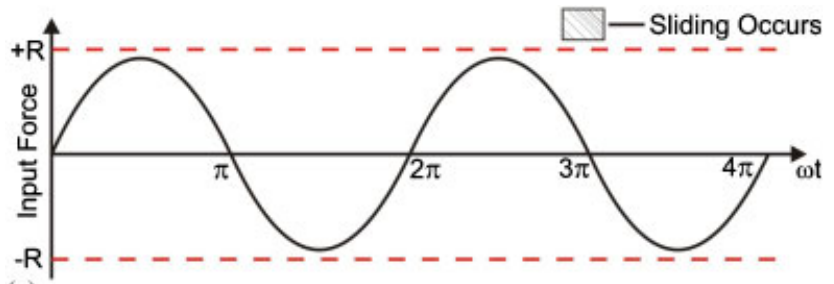

(a)
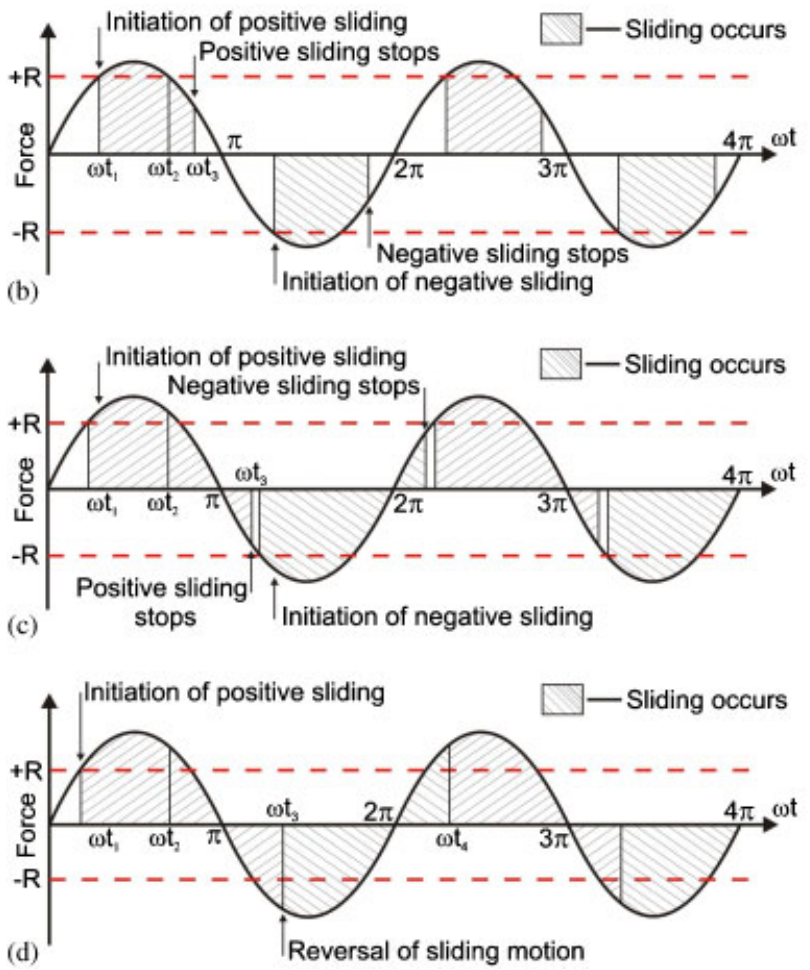

Figure 4. Representative diagrams for: (a) Case I; (b) Case II; (c) Case III; and (d) Case IV sliding behavior.

The acceleration, velocity, and displacement equations for the block, given as Equations (4)-(6), are valid until the block comes to rest at $t_{3}$.

Based on Equation (4), the block continues to accelerate until time $t_{2}$ at which point the input force is no longer greater than the friction force as can be seen in Figure 4(b-d). Equation (7) provides the calculation for time $t_{2}$. After time $t_{2}$ is reached, the block begins to decelerate and the subsequent motion is dependent on the time which it takes the block to come to rest or begin its reverse motion.

$$
\omega t_{2}=\pi-\arcsin \left(\frac{R}{F}\right)
$$


Case II is defined as the case in which the block stops sliding before $t=\pi / \omega$. The time at which the block stops sliding is labeled as $t_{3}$. As a result, Equations (4)-(6) are valid up until $t_{3}$ is reached. Since the block stops sliding before $t=\pi / \omega$, the block remains at rest until the input force can once again overcome the friction force. At this point, the input force has reversed directions as a result of the sinusoidal motion and the block slides in the opposite direction. Since the block starts once again from rest, the motion of the block is the same except in the opposite direction due to the input force now being $-F \sin (\omega t)$. Thus, the block will always slide between zero and the maximum relative displacement throughout the whole input motion with the initial half cycle of the sinusoidal input force causing the block to undergo its maximum relative displacement and the second half of the cycle causing a return to the initial starting point of the block as can be seen in Figure 4(b). In order for Case II to occur, the upper limit of $\omega t_{3}$ is $\pi$, since the block must stop before the input sinusoidal wave reverses its motion. By setting Equation (5) equal to zero and substituting the value of $\pi$ into the equation for $\omega t$, the $R / F$ ratio at which Case II occurs can be determined. As a result, Case II motion occurs if $0.725 \leqslant R / F<1$.

\subsection{Case III: equal sliding with residual displacement}

Case III is defined as the case in which the block stops sliding between the point where the input force reverses direction $(\omega t=\pi)$ and the point where the input force equals the friction force in the opposite direction $(\omega t=\pi+\arcsin (R / F))$. A schematic of the motion associated with Case III can be seen in Figure 4(c). Since the initial sliding motion for Case III stops before $\omega t$ equals $\pi$ $+\arcsin (R / F)$, Equations (4)-(6) remain valid. As with Case II motion, when the block comes to rest, it remains at rest until the friction force is overcome in the opposite direction. At this point, the block will experience the same motion as the initial sliding except in the opposite direction. The difference between Case II and Case III occurs when the input motion is terminated (at $\omega t$ equals $4 \pi$ in Figure 4(c)). Since the movement associated with Case III motion does not terminate within a half cycle of the sinusoidal input force, as occurs with Case II motion, the block cannot return to its initial position if the sinusoidal input force is terminated after a full cycle. It is found that Case III motion occurs if $0.537 \leqslant R / F<0.725$, by setting the upper limit of $\omega t_{3}$ equal to $\pi \arcsin (R / F)$.

\subsection{Case IV: progressive movement in a single direction}

The final possible sliding motion occurs when the block does not stop before the sinusoidal input motion in the opposite direction overcomes the friction force $\left(\omega t_{3}>\pi+\arcsin (R / F)\right)$. This motion is labeled as Case IV and is significantly more complicated than the previous three, since the motion in each direction now becomes unsymmetrical. A schematic of the motion associated with Case IV can be seen in Figure 4(d). The initial sliding motion for Case IV continues to be defined by Equations (4)-(6).

As a result of the block continuing its initial slide past $\omega t=\pi+\arcsin (R / F)$, the block will never come to a complete rest. Rather, upon reaching zero velocity, the block immediately begins to displace in the opposite direction. It is important to define the point at which this occurs. This can be done by solving Equation (8) for $\omega t_{3}$ where the following equation was obtained by setting Equation (5) equal to zero:

$$
\cos \left(\omega t_{3}\right)-\cos \left(\omega t_{1}\right)=-\frac{R}{F}\left(\omega t_{3}-\omega t_{1}\right) \quad \text { for }\left[\omega t_{3}>\pi+\arcsin (R / F)\right]
$$


By taking into account the change in direction of the friction force, the resulting relative acceleration, relative velocity, and relative displacement Equations (9)-(11) for the reverse motion can be defined. The initial conditions are defined as $\ddot{y}_{2}\left(t_{3}\right)=0, \dot{y}_{2}\left(t_{3}\right)=0$, and $y_{2}\left(t_{3}\right)=y_{1}\left(t_{3}\right)$.

$$
\begin{aligned}
\ddot{y}_{2}(t)= & \frac{F}{m} \sin (\omega t)+\frac{R}{m} \text { for }\left[\omega t_{3}<\omega t<\omega t_{4}\right] \\
\dot{y}_{2}(t)= & \frac{-F}{m \omega}\left[\left[\cos (\omega t)-\cos \left(\omega t_{3}\right)\right]-\frac{R}{F}\left(\omega t-\omega t_{3}\right)\right] \text { for }\left[\omega t_{3}<\omega t<\omega t_{4}\right] \\
y_{2}(t)= & \frac{F}{m \omega^{2}}\left\{\begin{array}{c}
-\left[\sin (\omega t)-\sin \left(\omega t_{3}\right)\right]+\frac{R}{2 F}\left[(\omega t)^{2}-\left(\omega t_{3}\right)^{2}\right] \cdots \\
+\cos \left(\omega t_{3}\right) \cdot\left(\omega t-\omega t_{3}\right)-\frac{R}{F} \omega t_{3}\left(\omega t-\omega t_{3}\right)
\end{array}\right\}+y_{1}\left(t_{3}\right) \\
& \text { for }\left[\omega t_{3}<\omega t<\omega t_{4}\right]
\end{aligned}
$$

For these equations, $t_{4}$ is defined as the time in which the block stops moving in the reverse directions. It should be noted that Equations (9)-(11) are similar in form to Equations (4)-(6), but account for the change in sign associated with the friction force.

By setting Equation (10) equal to zero, it can be assured that the block does not stop sliding until $\omega t_{4}>2 \pi+\arcsin (R / F)$. The resulting behavior is an unsymmetrical motion with the forward displacement always being slightly greater than the reverse displacement causing an overall increase in the residual relative displacement of the block during continued cycling. Case IV can be defined with respect to the $R / F$ ratio and occurs when $0<R / F<0.537$.

\subsection{Verification of theoretical formulation}

The theoretical formulation shows the importance of the friction force to input force ratio, $R / F$, and provides significant insight into the sliding behavior and sliding mechanism. Numerical analysis of the sliding block problem was undertaken in order to provide a sound solution in which to compare the theoretical results and a more flexible means to examine and expand upon the experimental results. The numerical analysis considered a single degree-of-freedom system where the nonlinear response of the system was solved for using Newmark's average acceleration method [24].

A bilinear model without strain hardening was adopted to represent the friction behavior. Given the previous assumption that the friction coefficient was constant, no distinction was made between the static and dynamic friction coefficients. A large stiffness was assigned to the elastic range and a small time step was used for the numerical analysis. Given that the system had a total mass of approximately $8360 \mathrm{~kg}$ and in light of the $5 \mathrm{~Hz}$ response frequency adopted in the test, the equivalent stiffness of the system was estimated to be $8.2 \times 10^{6} \mathrm{~N} / \mathrm{m}$. The elastic stiffness adopted for the analysis was approximately $1 \times 10^{13}$ times larger than this value, which was more than sufficient to assume that the system was rigid prior to slip. A time step of $\frac{1}{100}$ of the natural period $(0.002 \mathrm{~s}$ for the $5 \mathrm{~Hz}$ loading rate) was considered acceptable for the numerical studies. A smaller time step of $0.0005 \mathrm{~s}$ was used for this study to further ensure accuracy, particularly at points where the motion reverses or the sliding comes to a stop. 


\subsection{Comparison of analytical and numerical results}

A comparison of the sliding behavior obtained using both the theoretical and numerical approaches for Cases II-IV motion was undertaken to verify the theoretical formulation. The corresponding friction force to input force ratios, $R / F$, chosen for this task were $0.80,0.54$, and 0.20 , respectively. The natural frequency, $2 \pi$, and mass, $1.0 \mathrm{~kg}$, were kept constant for all cases. The displacement time history results for all three cases are shown in Figure 5 compared with those obtained using the theoretical equations presented previously. It is clear from the displacement time history results that the theoretical formulation for all three cases is accurate and reliable. Some slight deviations occur because of numerical errors associated with the finite integration time interval.

\section{EVALUATION OF THE EXPERIMENTAL SLIDING BEHAVIOR}

To further study the sliding behavior of steel on mortar, the previous experimental results are compared with numerical analyses in which the effects of non-ideal conditions with the input motion were considered. The $9 \mathrm{~m} / \mathrm{s}^{2}$ magnitude sinusoidal input accelerations are considered for all three loading frequencies. For all cases, the shake table acceleration time history recorded during the experimental study is used as the input motion for the numerical analysis to provide a more accurate comparison of the results. A second study compares experimental and numerical results for a non-stationary input motion.

\subsection{Sine wave input comparison}

Figure 6 provides the relative displacement time history results for the $9 \mathrm{~m} / \mathrm{s}^{2}$ magnitude sinusoidal acceleration input with loading frequencies of 1,2 , and $5 \mathrm{~Hz}$, respectively. For each loading, the experimental results are compared with the numerical results when the friction coefficient is taken as $0.70,0.725,0.75,0.775$, and 0.8 . For the $1 \mathrm{~Hz}$ loading frequency, the numerical results for all considered friction coefficients are provided in Figure 6 where for the 2 and $5 \mathrm{~Hz}$ loading frequencies only those numerical results which were closest to the experimental results are provided for the sake of clarity. In general, the numerical results followed a similar trend as the experimental results showing Case IV sliding behavior with an accumulation of residual displacement during cycling. When a friction coefficient between 0.75 and 0.775 for the 1 and $2 \mathrm{~Hz}$ loadings and 0.8 for the $5 \mathrm{~Hz}$ loading is adopted, the closest match between the experimental and numerical results is obtained. For all loading rates, there remain some minor differences between the numerical and experimental results. One means of increasing the correlation between the experimental and numerical results is to account for the minor non-symmetry of the friction behavior shown in Figure 2. The numerical results from all three loading rates suggest that the displacement time history is sensitive to the choice of the friction coefficient. However, the range of friction coefficients, which best predict the experimental behavior, is reasonable given the average friction coefficient of 0.78 obtained from the experimental results.

Based on the experimental specimen's properties, the sliding behavior should either be Case II or Case III (i.e. no increase in residual relative displacement) depending on the magnitude of the input acceleration. The stop and start behavior associated with Case II and Case III is clearly seen in Figure 6. However, there is also an accumulation of residual relative displacement. This 

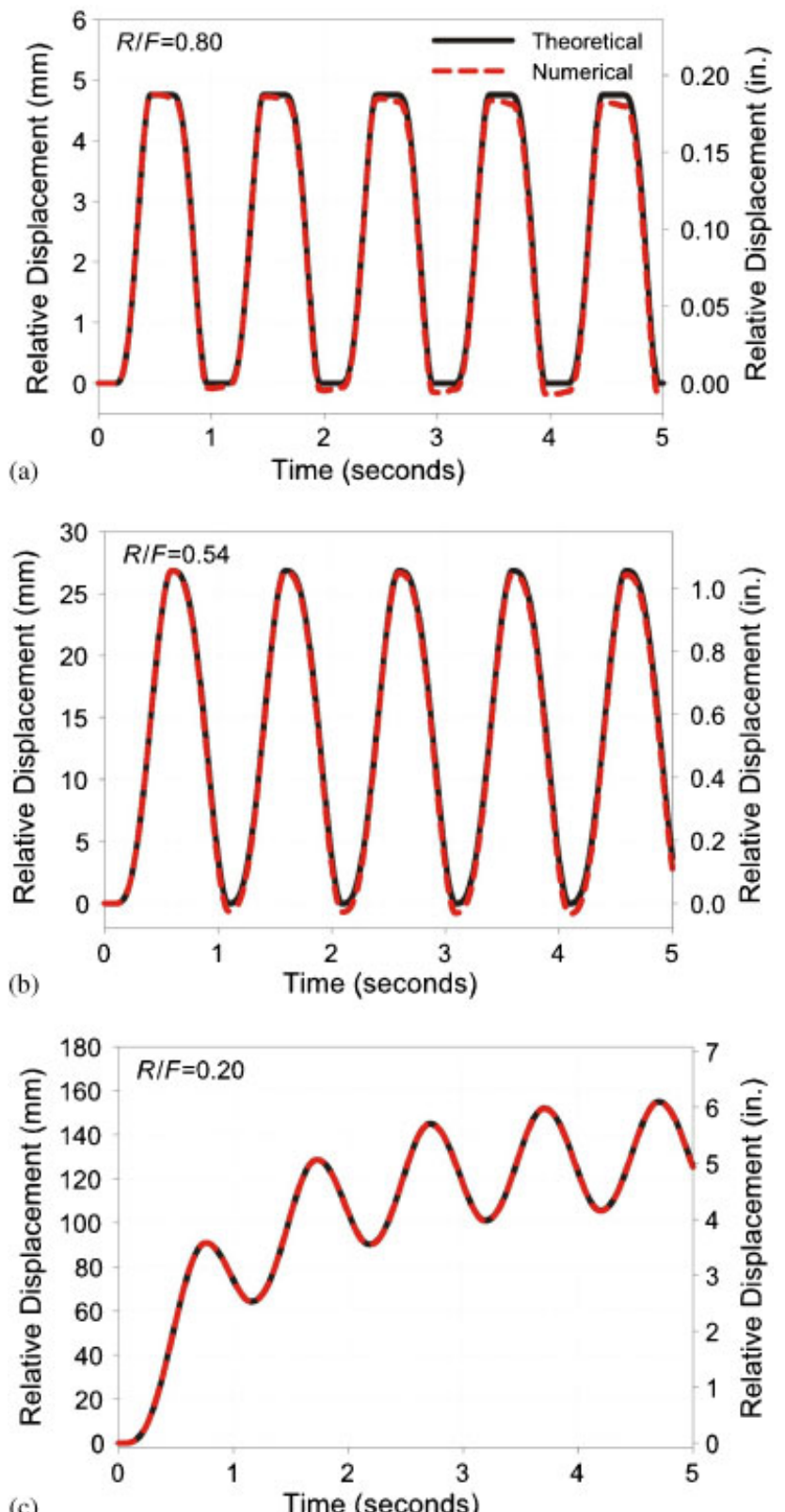

Figure 5. Comparison of the theoretical and numerical results for: (a) Case II;

(b) Case III; and (c) Case IV sliding behavior.

phenomenon is a result of the unsymmetrical nature of the input motion associated with the shake table test. As a result, the numerical analysis is able to capture this behavior when the actual table accelerations are used for the input motion. 


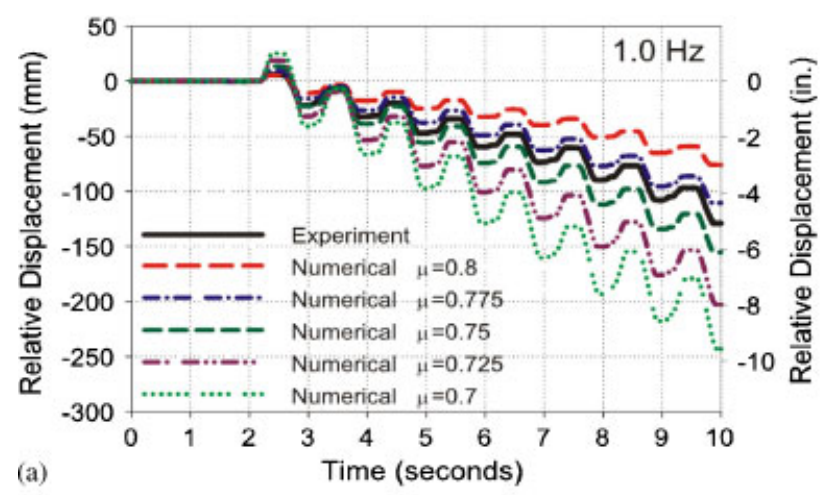

(a)
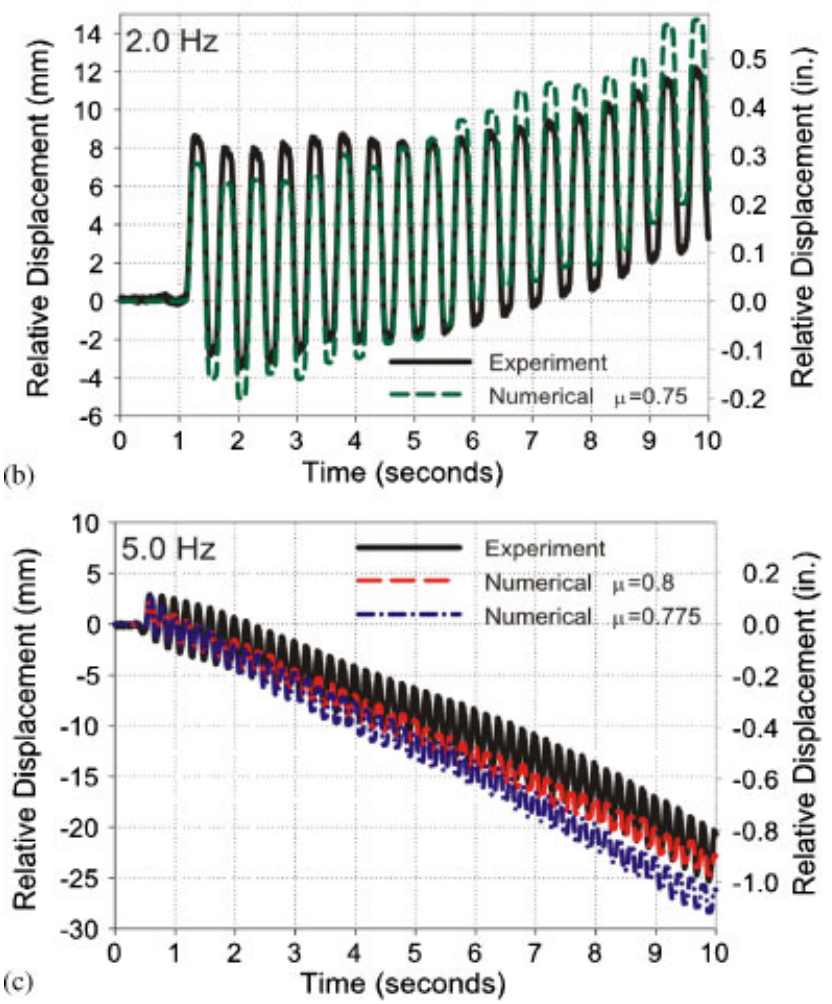

Figure 6. Experimental and numerical results for the $9 \mathrm{~m} / \mathrm{s}^{2}$ sine wave horizontal sliding at: (a) $1 \mathrm{~Hz}$; (b) $2 \mathrm{~Hz}$; and (c) $5 \mathrm{~Hz}$.

\subsection{Non-stationary input comparison}

One of the ultimate goals of this study is to determine the consistency of the friction coefficient during cycling. To more completely explore this phenomenon, the sliding behavior is considered under non-stationary motion as well. The horizontal component of the JMA Kobe ground motion 

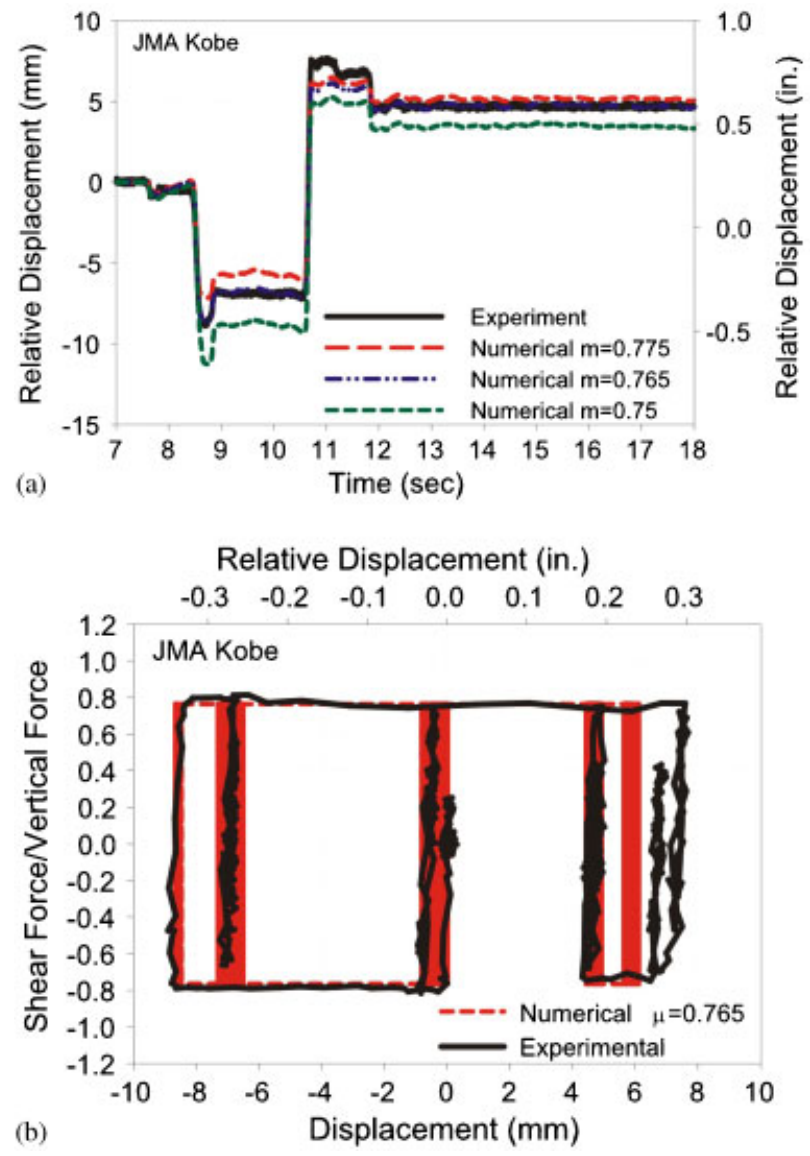

Figure 7. Experimental and numerical results for the horizontal-only JMA Kobe input motion: (a) relative displacement time history and (b) friction coefficient versus relative displacement.

[25] measured during the 1995 Hyogoken-Nanbu Earthquake in Japan was chosen as the input to represent a case of non-stationary motion considering a peak ground acceleration of $1.3 \mathrm{~g}$.

As with the sinusoidal input motion, the experimental results are compared with numerical analyses considering several different friction coefficients. The results of these tests are shown in Figure 7(a). A large relative displacement in the negative direction followed by an even larger relative displacement in the positive direction can be seen in the experimental results causing a residual relative displacement of approximately $4.7 \mathrm{~mm}$. The maximum relative displacements measured in the negative and positive direction were -8.9 and $7.9 \mathrm{~mm}$, respectively. As with the sinusoidal input accelerations, the numerical response is highly dependent on the choice of friction coefficient; however, the general behavior found during the experiment is obtained with numerical analysis. Using a friction coefficient of 0.765 for the numerical analysis, the maximum negative relative displacement and residual displacement were accurately simulated with only a small under prediction of $2 \mathrm{~mm}$ for the maximum positive relative displacement. 
Figure 7(b) provides the friction coefficient versus relative displacement comparison considering a friction coefficient of 0.765 . The friction coefficient remained very stable even during the nonstationary motion. Only a small decrease in the friction coefficient is observed for the long positive slip segment. This decrease may be associated with the difference between the static and dynamic friction coefficient resulting from changes in surface temperature and/or particle accumulation during extended periods of slip [26]. These results suggest that the difference between the static coefficient and dynamic friction coefficient may be more pronounced for longer slip distances associated with large input accelerations. The effects of larger slip distances and velocities are a subject of further study by the author's group and the details will be presented in the future.

\section{EFFECT OF VERTICAL ACCELERATION ON THE SLIDING BEHAVIOR}

For an actual structure, varying normal forces would be expected due to both vertical motion and overturning. As a result, it is important to examine how the friction coefficient also may vary. Since the test specimen is very low and flat, it is difficult to produce significant variations in the axial force due to overturning; hence, vertical motion was applied to explore the effect of varying axial force.

The test setup for the vertical acceleration study remained the same as that used for the horizontalonly acceleration study (Figure 1) with the exception of the acceleration input. Both horizontal and vertical sinusoidal acceleration inputs were used with magnitudes of 6 and $3 \mathrm{~m} / \mathrm{s}^{2}$, respectively. In order to simulate a typical ground motion, the magnitude of the vertical acceleration input was kept below that of the horizontal acceleration input. The difference in the frequency content between the horizontal and vertical motion was also taken as a parameter since this would determine whether the peaks of each input motion would coincide. To look at the bounds of the problem, the first test considered the case where the input frequencies were both $1 \mathrm{~Hz}$ and in-phase, whereas the second test considered differing frequencies where the horizontal sinusoidal input acceleration was run at $1 \mathrm{~Hz}$ and the vertical sinusoidal input acceleration was run at $5 \mathrm{~Hz}$.

The relative displacement time histories and friction coefficient versus relative displacement results for both tests are given in Figure 8. Comparing the relative displacement results, there is an obvious effect associated with the offset frequencies. For the test where the input frequencies were both $1 \mathrm{~Hz}$, Figure $8(\mathrm{a})$, the residual relative displacement was approximately $158 \mathrm{~mm}$ with sliding only occurring in one direction. This can be attributed to the fact that the vertical acceleration reduced the friction force at the same time the horizontal acceleration reached its maximum value in the negative direction. While for motion in the opposite direction, the vertical acceleration increased the friction force and did not allow sliding to occur even when the horizontal acceleration reached its maximum value. For the second test where the vertical and horizontal input frequencies differed, Figure 8(c), sliding occurred in both directions and a reduced residual relative displacement of approximately $15 \mathrm{~mm}$ was seen. For this case, the $5 \mathrm{~Hz}$ vertical acceleration allowed for sliding in both directions. For the test with the same input frequencies, the measured axial force fluctuated between 5.8 and $11.6 \mathrm{kN}$, whereas for the offset input frequencies the measured axial force fluctuated between 5 and $12 \mathrm{kN}$. In general, much smaller axial force fluctuations were seen during the horizontal-only input acceleration tests.

The friction coefficient versus relative displacement plot for the $1 \mathrm{~Hz}$ frequency test given in Figure 8(b) clearly shows that the horizontal forces were not large enough to overcome the friction coefficient for sliding to occur in the positive direction. Conversely, Figure 8(d) depicting the 

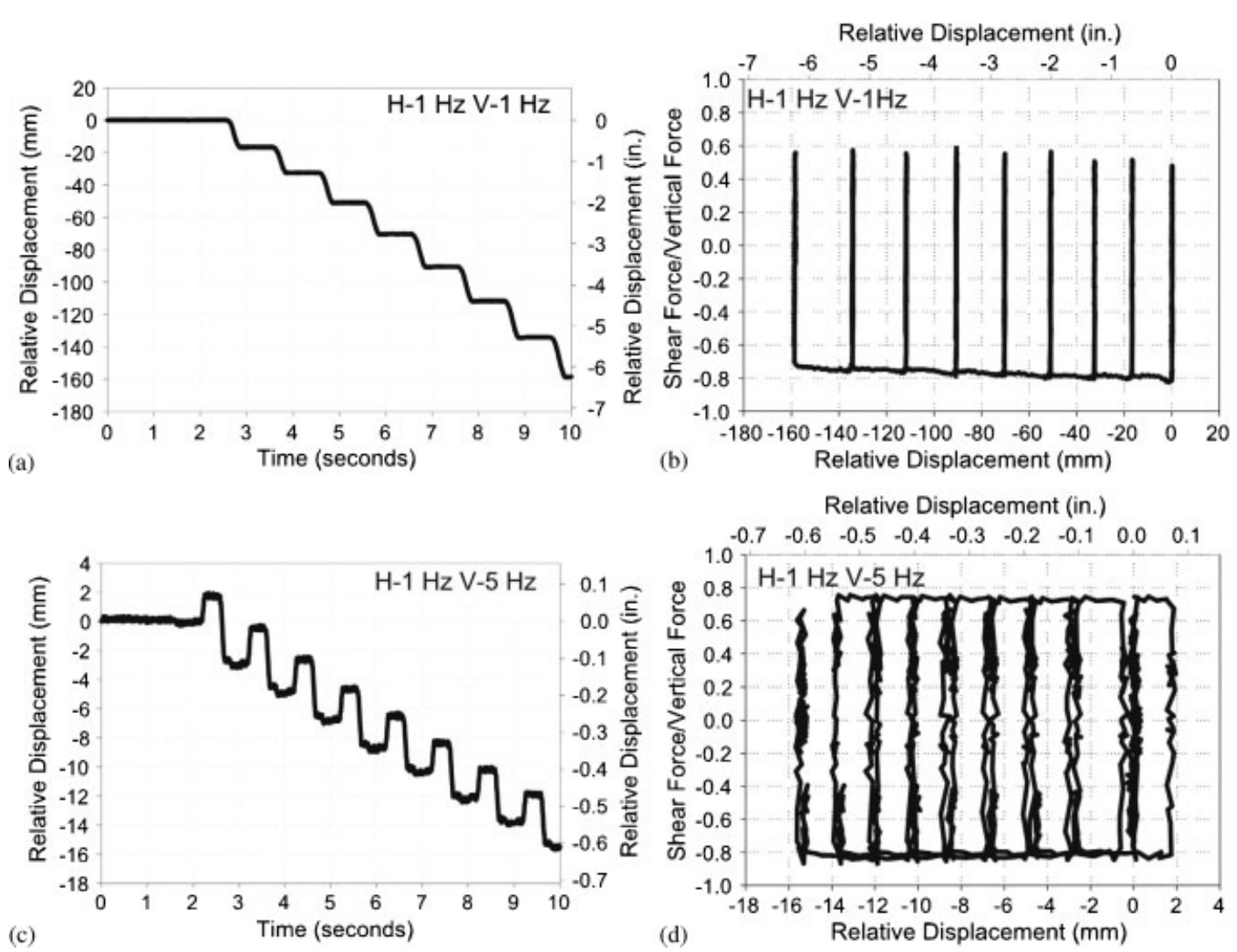

Figure 8. (a, c) Relative displacement time history and (b, d) friction coefficient versus relative displacement results considering both horizontal and vertical input accelerations.

hysteretic behavior for the offset frequency tests showed a similar behavior for the horizontal-only tests. For both tests, the average friction coefficient was approximately 0.78 . Only relatively small changes in the friction coefficient were observed during cycling for the same frequency case and with the positive versus negative sliding direction for the offset frequency case. However, for all cases, the friction coefficient remained between 0.74 and 0.84 .

\section{CONCLUSION}

The friction sliding behavior between steel and mortar was explored through experimental, theoretical, and numerical methods in order to consider how these two materials may interact during a seismic event. The shake table study used a rigid specimen with four contact points to evaluate changes in the friction resistance due to a horizontal-only sinusoidal acceleration input at various acceleration magnitudes and frequencies. The resulting sliding behavior was compared with the theoretical solution which was formulated considering an idealized rigid block sliding on a surface undergoing a sinusoidal acceleration. The theoretical solution showed that the sliding behavior 
was dependent on the ratio of the friction force to the input force. Numerical analysis was used to evaluate the overall sliding behavior and slip associated with individual cycles for various friction coefficients with respect to the experimental results. In considering the effects of non-stationary motion, a second set of experimental tests were conducted where both ground motion input accelerations and vertical accelerations were applied to the experimental specimen. The major findings from this study are detailed below.

- The experimental shake table study showed that after initial sliding the friction coefficient was approximately 0.78 . This friction coefficient value was maintained even during many repeated cycles using the same steel and mortar contact surface and was unaffected by the loading frequencies and acceleration magnitudes used for this study. The stability of the measured friction coefficient suggests the possibility of improving column base design to include multiple means of shear resistance for seismic applications in the future, such as superimposing the friction resistance with the shear strength of the anchor bolts. However, the value of the friction coefficient may deviate with differing steel surface conditions and mortar types. Work is being done to further consider these factors.

- The theoretical solution shows four possible sliding cases based on the ratio of the friction force to the input force. Cases I-IV represent no sliding, equal sliding in both directions, equal sliding with residual displacement, and progressive movement in a single direction, respectively, providing a means to better capture the nature and governing mechanisms of the sliding behavior.

- Under non-stationary motion, the friction coefficient remained relatively unchanged during sliding, suggesting the applicability of considering the friction behavior for seismic applications.

- The addition of vertical accelerations can have a significant effect on the overall relative displacement of the system due to the variations in the normal force at the point of contact. The effect that the addition of vertical acceleration has on the system is dependent on the respective amplitudes and phases of the horizontal and vertical input motions along with the friction coefficient. In general, the friction coefficient remained unchanged during cycling even when considering vertical accelerations. Along with vertical accelerations, it should also be kept in mind that overturning will provide another source of varying normal force at the column base.

\section{ACKNOWLEDGEMENTS}

This study was conducted as part of a research project supported by Grant-in-Aid for Scientific Research (Basic Research Category YB, 18760419) and administered by the Japan Society for the Promotion of Science. T. Nagae was the principal investigator on this project. P.-C. Zhang was supported by the Chinese Government Supported Researchers Program. J. McCormick was supported by the Japan Society for the Promotion of Science Postdoctoral Fellowship for Foreign Researchers Program.

\section{REFERENCES}

1. Kurata M, Nakashima M, Suita K. Effect of column base behavior on the seismic response of steel moment frames. Journal of Earthquake Engineering 2005; 9:415-438.

2. Nakashima M, Liu D. Instability and complete failure of steel columns subjected to cyclic loading. Journal of Engineering Mechanics (ASCE) 2005; 131(6):559-567.

3. Galambos TV. Guide to Stability Design Criteria for Metal Structures (5th edn). Wiley: New York, 1998. 
4. Wang T, McCormick J, Yoshitake N, Pan P, Murata Y, Nakashima M. Collapse simulation of a four-story steel moment frame by a distributed online hybrid test. Earthquake Engineering and Structural Dynamics 2008; 37:955-974.

5. Fisher JM, Kloiber LA. Steel Design Guide 1: Base Plate and Anchor Rod Design (2nd edn). American Institute of Steel Construction: Chicago, 2006.

6. American Institute of Steel Construction (AISC). Seismic Design Manual (13th edn). American Institute of Steel Construction: Chicago, 2006.

7. Architectural Institute of Japan (AIJ). Design Guidelines for Connections of Steel Building Structures. Architectural Institute of Japan: Japan, 2004.

8. Younis CJ, Tadjbakhsh IG. Response of sliding rigid structure to base excitation. Journal of Engineering Mechanics (ASCE) $1984 ; 110(3): 417-432$.

9. Shenton III HW. Criteria for initiation of slide, rock, and slide-rock rigid-body modes. Journal of Engineering Mechanics (ASCE) 1996; 122(7):690-693.

10. Taniguchi T, Miwa T. A simple procedure to approximate slip displacement of freestanding rigid body subjected to earthquake motions. Earthquake Engineering and Structural Dynamics 2007; 36:481-501.

11. Tung CC. Initiation of motion of a free-standing body to base excitation. Earthquake Engineering and Structural Dynamics 2007; 36:1431-1439.

12. Konstantinidis D, Makris N. Experimental and analytical studies on the response of freestanding laboratory equipment to earthquake shaking. Earthquake Engineering and Structural Dynamics 2008; DOI: 10.1002/eqe.871. online early view.

13. Bondonet G, Filiatrault A. Frictional response of PTFE sliding bearing at high frequencies. Journal of Bridge Engineering (ASCE) 1997; 2(4):139-148.

14. Iemura H, Taghikhany T, Takahashi Y, Jain SK. Effect of variation of normal force on seismic performance of resilient sliding isolation systems in highway bridges. Earthquake Engineering and Structural Dynamics 2005; 34:1777-1797.

15. Roussis PC, Constantinou MC. Experimental and analytical studies of structures seismically isolated with an uplift-restraining friction pendulum system. Earthquake Engineering and Structural Dynamics 2006; 35:595-611.

16. Fenz DM, Constantinou MC. Behaviour of the double concave friction pendulum bearing. Earthquake Engineering and Structural Dynamics 2006; 36:1403-1424.

17. Tsai CS, Chen W-S, Chiang T-C, Chen B-J. Component and shaking table tests for full-scale multiple friction pendulum system. Earthquake Engineering and Structural Dynamics 2006; 35:1653-1675.

18. Jangid RS. Stochastic response of bridges seismically isolated by friction pendulum system. Journal of Bridge Engineering 2008; 13(4):319-330.

19. Pall AS, Marsh C, Fazio P. Friction joints for seismic control of large panel structures. Journal of Prestressed Concrete Institute $1980 ; 25(6): 38-61$.

20. Larson DS, Fafitis A. Slip-stick steady-state solution for simple coulomb-damped mass. Journal of Engineering Mechanics (ASCE) 1995; 121(2):289-298.

21. Larson DS, Fafitis A. Periodic response and stability of rigid mass resting on friction-damped SDOF oscillator. Journal of Engineering Mechanics (ASCE) 1995; 121(11):1226-1233.

22. Ng C-L, Xu Y-L. Seismic response control of a building complex utilizing passive friction damper: experimental investigation. Earthquake Engineering and Structural Dynamics 2006; 35:657-677.

23. Nagae T, Ikenaga M, Nakashima M, Suita K. Shear friction between base plate and mortar in exposed steel column base. Transactions of AIJ Journal of Structural and Construction Engineering 2005; 606(8):217-223 (in Japanese).

24. Newmark NM. A method of computation for structural dynamics. Journal of the Engineering Mechanics Division (ASCE) 1959; 85:67-94.

25. Yamaguchi N, Yamazaki F. Estimation of strong motion distribution in the 1995 Kobe earthquake based on building damage data. Earthquake Engineering and Structural Dynamics 2001; 30:787-801.

26. Christopoulos C, Filiatrault A. Principles of Passive Supplemental Damping and Seismic Isolation. IUSS Press: Pavia, Italy, 2006. 